\title{
Changements historiques à long terme du débit écoulé dans le Haut bassin du fleuve Sénégal
}

\author{
Cheikh Faye \\ Département de Géographie, U.F.R. Sciences et Technologies, Université Assane Seck de Ziguinchor, \\ Laboratoire de Géomatique et d'Environnement, BP 523 Ziguinchor, Sénégal \\ Correspondence: Cheikh Faye (cheikh.faye@univ-zig.sn) \\ Published: 16 November 2021
}

\begin{abstract}
Résumé. In this study, the flow trends on either side of the Manantali dam, in the upper Senegal River basin, were assessed. The assessment was carried out for the period 1970 to 2019 using the Sen slope and the MannKendall test. The Mann-Kendall trend test was used to indicate the direction of the trends, while the Sen slope was used to assess the magnitude of the change. The results of the study showed that there was an upward trend in the flow of the Senegal River both annually and for every month upstream (Bafing-Makana station) and downstream (Bakel station) from the barrage. The strongest upward trend is noted in October on the upstream station with $4.12 \mathrm{~m}^{3} /$ month and in September on the downstream station with $10.99 \mathrm{~m}^{3} /$ month. In return, the weakest upward trend is observed in May on the upstream station with $0.07 \mathrm{~m}^{3} / \mathrm{month}$ and in August on the downstream station with $0.67 \mathrm{~m}^{3} /$ month. The annual flow also showed a significant increase upstream (with a rate of $0.35 \mathrm{~m}^{3} /$ year) and downstream (with a rate of $4.53 \mathrm{~m}^{3} /$ year) with a confidence level of $99 \%$. The results of the study also showed that an increase in precipitation causes an increase in runoff, despite the construction of hydraulic structures.
\end{abstract}

\section{Introduction}

Les fortes concentrations de gaz à effet de serre provoquent une augmentation de la température globale (Crawford et al., 2019). L'augmentation de la température joue un rôle défavorable dans la modification des composants du cycle hydrologique global (Ahmed et al., 2019). Les précipitations sont considérées comme la composante la plus vigoureuse du cycle hydrologique mondial, qui aurait changé dans plusieurs régions du monde (Asfaw et al., 2018). L'altération des caractéristiques des précipitations provoque de graves conséquences sur la société sous la forme d'inondations et de sécheresses, ce qui peut avoir un impact négatif sur la situation socioéconomique des populations (Ezzine et al., 2014). Les modifications du débit des cours d'eau ou des rivières sont souvent associées aux modifications des précipitations (Rawshan et al., 2019).

Les évaluations de tendance du débit des fleuves fournissent des informations de base relatives à l'eau (Gajbhiye et al., 2016). Le Sénégal, situé en Afrique tropicale, a également connu des changements dans les précipitations et la température au cours des cinquante dernières années (Faye et al., 2019). Devant la succession d'épisodes climatologiques (sécheresses et inondations) et hydrologiques (crues et étiages) extrêmes, les études faites sur le bassin versant du fleuve Sénégal ont donc procédé à une analyse des données pour caractériser le changement climatique dans ce bassin. Sur la période la plus récente, certaines études ont mis en exergue la hausse de la pluie et de l'écoulement dans la zone depuis les années 2000 et qui présage d'une amélioration du régime hydrologique (Ali et al., 2008) et une recrudescences des inondations. La connaissance de l'évolution du débit des cours d'eau au Sénégal est souvent limitée en raison de la disponibilité et de la qualité des données. La présente étude a examiné les méthodes non paramétriques pour caractériser les tendances et leur signification des cours d'eau à partir de données historiques. L'évaluation a été réalisée sur des données de cinquante années allant de 1970 à 2019. 


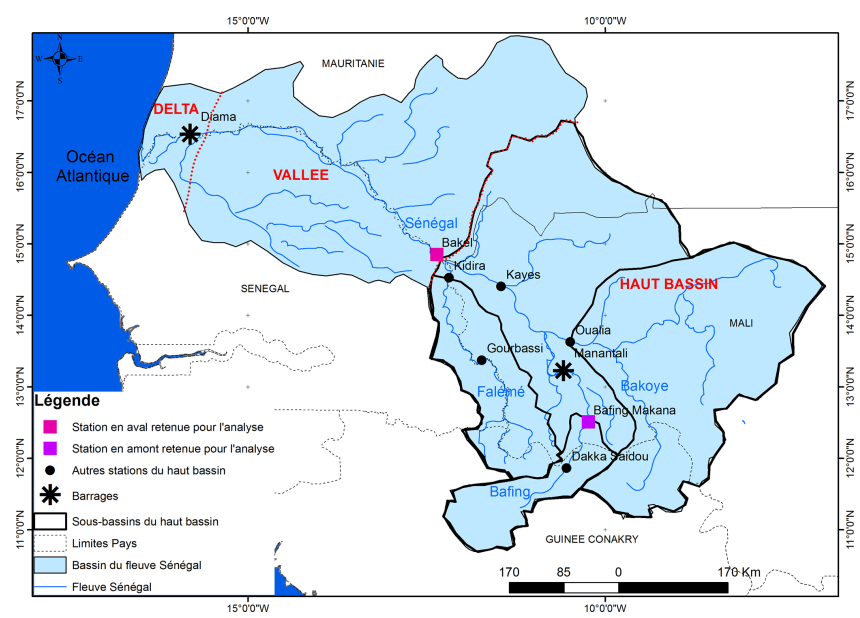

Figure 1. Localisation du bassin versant du fleuve Sénégal et de son haut bassin.

\section{Zone d'étude}

Le fleuve Sénégal, long de quelques $1700 \mathrm{~km}$, draine un bassin de $300000 \mathrm{~km}^{2}$, à cheval sur quatre pays que sont, d'amont en aval, la Guinée, le Mali, le Sénégal et la Mauritanie (Fig. 1). Il va de $10^{\circ} 20^{\prime}$ à $17^{\circ} \mathrm{N}$ et de $7^{\circ}$ à $12^{\circ} 20^{\prime} \mathrm{W}$ et est formé de plusieurs affluents, dont les principaux sont le Bafing, le Bakoye et la Falémé qui prennent leurs sources en Guinée et forment le haut bassin (OMVS/Projet FEM/ BFS, 2008) (Fig. 1). Le fleuve Sénégal ainsi formé par la jonction entre le Bafing et le Bakoye, reçoit la Kolimbiné puis le Karokoro sur la droite et la Falémé sur la gauche, à $50 \mathrm{~km}$ en amont de Bakel. Dans la partie Sud du bassin, la densité du réseau hydrographique découle de la nature imperméable des sols et de la faiblesse de l'infiltration (Michel, 1973; Rochette, 1974).

\section{Matériels et méthodes}

\subsection{Données}

La base de données de la station amont (Bafing-Makana) et celle aval (Bakel) sont retenues pour ladite étude. Les données hydrométriques ont été mises à notre disposition par l'Organisation pour la Mise en Valeur du Fleuve Sénégal (OMVS). Ces données concernent les débits journaliers, (de 1970 à 2019), à partir desquels sont calculés les débits annuels et saisonniers sont utilisées dans l'analyse.

\subsection{Méthodes}

\subsubsection{Analyse fréquentielle}

Dans la présente étude, les distributions gamma, celle de la valeur extrême généralisée et la distribution normale, qui sont largement utilisées dans de nombreuses études clima- tiques, ont été appliquées aux jeux de données du bassin du fleuve Sénégal.

\subsubsection{Estimation de l'ampleur du changement}

Dans cette étude, la méthode de l'estimateur des pentes de Sen (Sen, 1968) a été appliquée pour calculer l'intensité du changement du débit de la rivière. La méthode est bien connue et largement utilisée pour le calcul des tendances (Gajbhiye et al., 2016).

\subsubsection{Tests de tendance de Mann-Kendall}

Le test de Mann Kendall (Mann, 1945) est un test non paramétrique fréquemment utilisé pour évaluer des tendances dans les séries chronologiques. Le test est également approuvé par l'Organisation météorologique mondiale (OMM) pour les évaluations des tendances hydrométéorologiques.

\section{Résultats et discussion}

\subsection{Analyse fréquentielle}

Les résultats obtenus sont indiqués dans le Tableau 1. Les valeurs en gras dans le Tableau indiquent que l'hypothèse nulle H0 : «absence de tendance dans la série » est rejetée au niveau de confiance de $95 \%$, tandis que les valeurs en italique indiquent le meilleur ajustement. A la station en amont (Bafing-Makana), il a été constaté que toutes les données suivaient la distribution gamma, tandis que l'hypothèse nulle avait été rejetée pour certains mois (juin à octobre), sur le module, sur le débit maximal et sur le débit des périodes de hautes et de basses eaux pour la distribution normale et GEV.

De même, pour la station en aval (Bakel), il a été constaté que toutes les données suivaient la distribution gamma. Dans la distribution généralisée des valeurs extrêmes, les mois de mars à septembre et de janvier à mars, le module, le débit maximal annuel et le débit des périodes de hautes et de basses eaux indiquaient une hypothèse nulle rejetée. De même, dans la distribution normale, l'hypothèse nulle rejetée sur les même variables (mois de mars à septembre et de janvier à mars, module, débit maximal annuel et débit des périodes de hautes et de basses eaux) en plus du mois d'octobre.

\subsection{Distribution saisonnière du débit}

Dans la partie amont barrage (Fig. 2), sur les deux périodes, on note une évolution similaire malgré des débits mensuels légèrement plus élevés sur la période 1988-2019. Les pics sont notés en septembre (avec $740 \mathrm{~m}^{3} / \mathrm{s}$ de 1970 à 1987 et $867 \mathrm{~m}^{3} / \mathrm{s}$ de 1988 à 2019) et les débits les plus faibles en avril en 1970-1987 (avec 3,8 $\mathrm{m}^{3} / \mathrm{s}$ ) et mai en 1988$2019\left(\right.$ avec $\left.5,9 \mathrm{~m}^{3} / \mathrm{s}\right)$. Les mois de la période de hautes eaux (juillet, août, septembre et octobre) affichaient les débits les 
Tableau 1. Statistiques de test de Kolmogorov-Smirnov obtenues lors de la répartition par ajustement du débit du fleuve Sénégal aux stations en amont (Bafing-Makana) et en aval (Bakel) du barrage.

\begin{tabular}{lrrr|rrr}
\hline \multirow{2}{*}{ Paramètres } & \multicolumn{3}{c|}{ Station en amont } & \multicolumn{3}{c}{ Station en aval } \\
\cline { 2 - 7 } & Gamma & GEV & Normale & Gamma & GEV & Normale \\
\hline Mai & 0,4287 & 0,3621 & 0,2297 & 0,4779 & 0,2466 & 0,1941 \\
Juin & 0,4183 & 0,0694 & 0,1631 & 0,4476 & 0,1100 & 0,1101 \\
Juillet & 0,4638 & 0,0737 & 0,1159 & 0,4717 & 0,0695 & 0,1270 \\
Août & 0,3907 & 0,1107 & 0,1143 & 0,5477 & 0,0869 & 0,1265 \\
Septembre & 0,4952 & 0,1125 & 0,1478 & 0,5078 & 0,1131 & 0,1052 \\
Octobre & 0,4166 & 0,0831 & 0,0933 & 0,5578 & 0,2996 & 0,1499 \\
Novembre & 0,5064 & 0,5165 & 0,2022 & 0,5144 & 0,7551 & 0,2028 \\
Décembre & 0,3534 & 0,2209 & 0,2052 & 0,4323 & 0,4774 & 0,2090 \\
Janvier & 0,3217 & 0,5292 & 0,2279 & 0,5338 & 0,1491 & 0,1676 \\
Février & 0,2990 & 0,5918 & 0,2444 & 0,4414 & 0,1144 & 0,1206 \\
Mars & 0,2883 & 0,4898 & 0,2055 & 0,4231 & 0,1654 & 0,1631 \\
Avril & 0,2907 & 0,5170 & 0,1645 & 0,4688 & 0,2225 & 0,2013 \\
Module & 0,3158 & 0,0997 & 0,1087 & 0,4808 & 0,0855 & 0,1256 \\
Débit Maximum & 0,4665 & 0,0618 & 0,0651 & 0,4897 & 0,0738 & 0,0758 \\
Débit minimum & 0,3711 & 0,6479 & 0,2338 & 0,4490 & 0,3125 & 0,2015 \\
Hautes eaux & 0,3884 & 0,1000 & 0,0938 & 0,5254 & 0,0650 & 0,1142 \\
Basses eaux & 0,3269 & 0,0695 & 0,1546 & 0,4474 & 0,1174 & 0,1099 \\
\hline
\end{tabular}
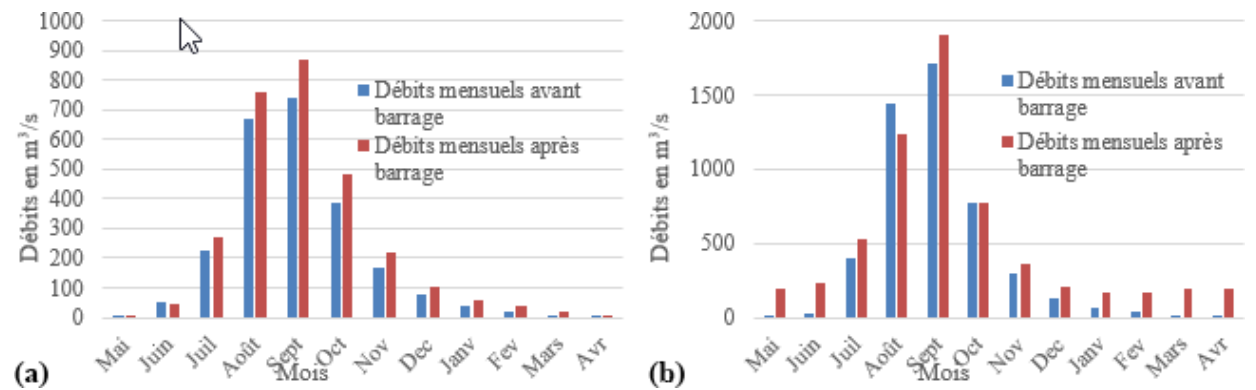

Figure 2. Distribution saisonnière du débit enregistré en amont (a) et en aval (b) du barrage de Manantali.

plus élevés sur les deux périodes. Sur la période de basses eaux (le reste de l'année), les débits étaient très faibles.

Dans la partie aval barrage (Fig. 2), l'évolution du débit montre une nette opposition entre la période 1970-1987 (dont l'évolution est assez semblable de celle notée à BafingMakana sur les deux périodes) et celle 1988-2019 qui a vu ses débits en période de basses eaux fortement augmentés (son débit minimal est plus de 90 fois plus important que celui de 1970-1987). Cette augmentation s'explique par la politique de soutien des étiages du barrage de Manantali. Toutefois, sur les deux périodes, les pics sont notés en septembre (avec $1714 \mathrm{~m}^{3} / \mathrm{s}$ en $1970-1987$ et $1902 \mathrm{~m}^{3} / \mathrm{s}$ en 1988 2019) et les débits les plus faibles en mai en 1970-1987 (avec $2,07 \mathrm{~m}^{3} / \mathrm{s}$ ) et en $1988-2019\left(\right.$ avec $\left.188 \mathrm{~m}^{3} / \mathrm{s}\right)$. Contrairement à la période avant barrage, sur la période après barrage, on peut noter que le débit a commencé à augmenter à partir du mois de janvier. Cette augmentation s'est opérée après une diminution allant de septembre à janvier, ce qui est en contradiction avec le débit avant le barrage, où il a commencé à diminuer à partir du mois de septembre jusqu'en avril. Les augmentations de débit sont dues au surplus d'eau du réservoir et au maintien d'un débit minimal dans le fleuve (Faye, 2015). Il ressort aussi de la Fig. 2 que le débit était très faible de décembre à mai en amont, alors qu'il s'élève sur cette période au niveau de la partie aval.

\subsection{Tendances des flux mensuels et annuels}

Le Tableau 2 présente l'importance de la statistique $Z$ (tau de Kendall) et la pente de Sen aux niveaux de confiance de $90 \%$ à $95 \%$ de chaque variable. Les résultats montrent des augmentations importantes des tendances des deux côtés du barrage (de toutes les variables, seul le mois de juin a connu une tendance à la baisse de son écoulement à la station de Bafing-Makana). En amont, les mois d'octobre à avril ont montré des tendances à la hausse significatives au niveau de confiance de $99 \%$. Les tendances de mai étaient significatives au niveau de confiance de $95 \%$, tandis que les ten- 
Tableau 2. Tendances des débits mensuels et moyens annuels des rivières obtenues à l'aide de la pente de Sen et du test de tendance de Mann-Kendall situé en amont et en aval du barrage de Manantal.

\begin{tabular}{|c|c|c|c|c|c|c|}
\hline \multirow[t]{2}{*}{ Paramètres } & \multicolumn{3}{|c|}{ Station en amont (Bafing Makana) } & \multicolumn{3}{|c|}{ Station en aval (Bakel } \\
\hline & Tau de Kendall & Significativité & Pente de Sen & Tau de Kendall & Significativité & Pente de Sen \\
\hline Mai & 0,2135 & $* *$ & 0,0712 & 0,6344 & $* * *$ & 6,9206 \\
\hline Juin & $-0,0034$ & & $-0,0077$ & 0,6361 & $* * *$ & 7,7636 \\
\hline Juillet & 0,1650 & * & 1,8607 & 0,3844 & $* * *$ & 5,6139 \\
\hline Août & 0,1480 & & 2,8723 & 0,0119 & & 0,6723 \\
\hline Septembre & 0,1361 & & 2,3319 & 0,1378 & & 10,9858 \\
\hline Octobre & 0,3180 & $* * *$ & 4,1147 & 0,0493 & & 1,5702 \\
\hline Novembre & 0,4286 & $* * *$ & 2,6147 & 0,1259 & & 1,5357 \\
\hline Décembre & 0,3861 & $* * *$ & 1,1942 & 0,2126 & $* *$ & 1,8892 \\
\hline Janvier & 0,4082 & $* * *$ & 0,7428 & 0,4337 & $* * *$ & 3,2915 \\
\hline Février & 0,4541 & $* * *$ & 0,5293 & 0,6020 & $* * *$ & 4,9282 \\
\hline Mars & 0,4507 & $* * *$ & 0,3203 & 0,6003 & $* * *$ & 5,9834 \\
\hline Avril & 0,4065 & $* * *$ & 0,1739 & 0,6344 & $* * *$ & 6,7668 \\
\hline Module & 0,3452 & $* * *$ & 1,821 & 0,2619 & $* * *$ & 4,531 \\
\hline Débit Maximum & 0,0255 & & 2,1833 & 0,0952 & & 10,8645 \\
\hline Débit minimum & 0,5352 & $* * *$ & 0,0709 & 0,6785 & $* * *$ & 3,9356 \\
\hline Hautes eaux & 0,2466 & $* *$ & 2,9862 & 0,0884 & & 4,3142 \\
\hline Basses eaux & 0,3384 & $* * *$ & 0,8891 & 0,5748 & $* * *$ & 5,3217 \\
\hline
\end{tabular}

* Significative à $90 \%, * *$ Significative à $95 \%$ et *** Significative à $99 \%$

dances de juillet étaient significatives au niveau de confiance de $90 \%$. Il est important de mentionner ici que le module, le débit minimal et le débit en période de basses eaux avaient également des tendances à la hausse significatives au niveau de confiance de $99 \%$. La tendance significative la plus élevée avec $4,11 \mathrm{~m}^{3} / \mathrm{an}$ a été observée pour le mois d'octobre, suivie du mois d'août avec $2,87 \mathrm{~m}^{3} / \mathrm{s} / \mathrm{an}$. Par ailleurs, la tendance du module a montré une variation de $1,82 \mathrm{~m}^{3} / \mathrm{s} / \mathrm{an}$ avec un niveau de confiance de $99 \%$. On peut également noter qu'un seul débit mensuel a montré une légère tendance à la baisse (le mois de juin avec $-0,0034 \mathrm{~m}^{3} / \mathrm{s} / \mathrm{an}$. Une augmentation des tendances du débit des cours d'eau a également été observée dans d'autres parties du monde (Soro et al., 2014).

Le barrage indique une différence du nombre de mois de hautes eaux (c'est-à-dire mois à CMD > 1). Sur la période 1970-1987, le nombre de mois de hautes eaux est de 3 mois, alors que sur la période 1988-2019, il est de 4 mois. Ainsi, la mise en eau du barrage de Manantali change le régime du bassin à la station de Bakel qui passe d'un régime fluvial tropical pur (avant barrage) à un régime fluvial tropical de transition (après barrage). De plus, le débit de la période de hautes eaux est passé de $506 \mathrm{~m}^{3} / \mathrm{s}$ (en 1970-1987) à $595 \mathrm{~m}^{3} / \mathrm{s}$ (en 1988-2019) dans la partie amont du bassin (soit une hausse de $17,6 \%$ ) et de $1085 \mathrm{~m}^{3} / \mathrm{s}$ (en 1970-1987) à $1109 \mathrm{~m}^{3} / \mathrm{s}$ (en 1988-2019) dans la partie aval du bassin (soit une faible hausse de $2,21 \%$ ).

\section{Conclusions}

Cette étude avait comme objectif de caractériser les tendances du débit de part et d'autre du barrage de Manantali, dans le haut bassin du fleuve Sénégal. L'évaluation a été réalisée sur la période de 1970 à 2019 avec la méthode d'estimateur de la pente de Sen et le test de Mann-Kendall. Les résultats de l'analyse des tendances ont montré que le débit du fleuve avait augmenté presque tous les mois des deux côtés du barrage. Le mois d'octobre a enregistré le taux de changement le plus élevé en amont, tandis que le mois de septembre a enregistré le taux de changement le plus élevé en aval du barrage. Les mois de mai et d'août ont montré les changements les plus faibles respectivement en amont et en aval du barrage. Des changements importants ont été remarqués aux mois de mai, juin, janvier, février et mars. De même, les modifications du débit annuel du fleuve ont également été jugées importantes. Des changements significatifs positifs ont également été observés d'abord dans les débits maximum et minimum et ensuite dans les débits en périodes de hautes et de basses eaux des deux côtés du barrage. Il a été constaté que le débit maximal avait augmenté de manière relativement significative du côté amont du barrage, ce qui indique une augmentation des précipitations. De plus, la construction de structures hydrauliques peut être une cause majeure de la hausse du débit en période d'étiages. Les résultats de cette étude devraient fournir une dcumentation importante à la planification et à la gestion de l'agriculture et des ressources en eau du bassin du fleuve Sénégal. 
Disponibilité des données. Aucun ensemble de données n'a été utilisé dans cet article.

Intérêts concurrents. The contact author has declared that there are no competing interests.

Clause de non-responsabilité. Publisher's note : Copernicus Publications remains neutral with regard to jurisdictional claims in published maps and institutional affiliations.

Déclaration du numéro spécial. This article is part of the special issue "Hydrology of Large River Basins of Africa". It is a result of the 4th International Conference on the "Hydrology of the Great Rivers of Africa", Cotonou, Benin, 13-20 November 2021.

Financement. This research has been supported by the Systematics Association.

\section{Références}

Ahmed, K., Shahid, S., Chung, E.-S., Wang, X.-J., and Harun S. B. : Climate Change Uncertainties in Seasonal Drought SeverityArea-Frequency Curves : Case of Arid Region of Pakistan, J. Hydrol., 570, 473-485, 2019.

Ali, A., Lebel, T. and Amami, A. : Signification et usage de l'indice pluviométrique au Sahel, Sécheresse, 19, 227-235, 2008.

Asfaw, A., Simane, B., Hassen, A., and Bantider, A. : Variability and time series trend analysis of rainfall and temperature in northcentral Ethiopia : A case study in Woleka sub-basin, Weather Clim. Extremes, 19, 29-41, 2018.

Crawford, J., Venkataraman, K., and Booth, J. : Developing climate model ensembles : A comparative case study, J. Hydrol., 568, $160-173,2019$.
Ezzine, H., Bouziane, A., and Ouazar, D. : Seasonal comparisons of meteorological and agricultural drought indices in Morocco using open short time-series data, Int. J. Appl. Earth Obs. Geoinf., 26, 36-48, 2014.

Faye, C. : Impact du changement climatique et du barrage de Manantali sur la dynamique du régime hydrologique du fleuve Sénégal à Bakel (1950-2014), Bulletin de la Société Géographique de Liège : BSGLg, 64, 69-82, 2015.

Faye, C., Grippa, M., and Wood, S. : Use of the Standardized Precipitation and Evapotranspiration Index (SPEI) from 1950 to 2018 to determine drought trends in the Senegalese territory, Climate Change, 5, 327-341, 2019.

Gajbhiye, S., Meshram, C., Singh, S. K., Srivastava, P. K., and Islam, T. : Precipitation trend analysis of Sindh River basin, India, from 102-year record (1901-2002), Atmos. Sci. Lett., 17, 71-77, 2016.

Michel, P. : Les bassins des fleuves Sénégal et Gambie : Etude géomorphologique, Mémoires ORSTOM n ${ }^{\circ}$ 63-3tomes, 752 pp., 1973.

Mann, H. B. : Non-Parametric Test against Trend, Econometrica, 13, 245-259, https://doi.org/10.2307/1907187, 1945.

OMVS, Projet FEM/Bassin du fleuve senegal : Plan d'Action Stratégique de Gestion des Problèmes Environnementaux Prioritaires du Bassin du Fleuve Sénégal, Version finale, 133 pp., 2008.

Rawshan, A., Arez, I., Arien, H., and Nadeem, N. : Long Term Historic Changes in the Flow of Lesser Zab River, Iraq, Hydrology, 6, 1-12, https://doi.org/10.3390/hydrology6010022, 2019.

Rochette, C. : Monographie hydrologique du fleuve Sénégal, Coll. Mém. ORSTOM, 1442 pp., 1974.

Sen, P. K. : Estimates of the regression coefficient based on Kendall's tau, J. Am. Stat. Assoc., 63, 1379-1389, 1968.

Soro, G. E., Anouman, D. G. L., Goula, B. I. T. A., Srohorou, B., and Savane, I. : Caractérisation des séquences de sécheresse météorologique a diverses échelles de temps en climat de type soudanais : cas de l'extrême Nord-ouest de la cote d'ivoire, Larhyss/Journal, 18, 107-124, 2014. 